\title{
Comparación de las propiedades físico-mecánicas del bambú Guadua angustifolia Kunth de diferentes municipios de Colombia
}

Comparison of physical and mechanical
properties of bamboo Guadua angustifolia Kunth
from different municipalities of Colombia

MSc. Arq. Laura C. Sánchez E.(1) MSc. Ing. Civil Andrés F. Guerra R.(1) MSc. Arq. Jorge E. Lozano P. (1)

(1) Universidad Nacional de Colombia Icsancheze@unal.edu.co afguerrari@unal.edu.co jelozanop@unal.edu.co

Recibido: 7 de Julio, 2019

Aceptado: 11 de Diciembre, 2019

Doi: http://dx.doi.org/10.24133/ciencia. v22i1.1289

"THIS IS AN OPEN ACCESS ARTICLE UNDER THE TERMS OF THE CREATIVE COMMONS ATTRIBUTION LICENSE, WHICH PERMITS USE, DISTRIBUTION AND REPRODUCTION IN ANY MEDIUM, PROVIDED THE ORIGINAL WORK IS PROPERLY CITED."

\begin{abstract}
RESUMEN
Esta investigación se realiza mediante ensayos físico-mecánicos para la caracterización del bambú Guadua angustifolia Kunth proveniente de los municipios de Socorro- Santander, Guaduas- Cundinamarca, Oiba -Santander y guadua proveniente del departamento del Tolima, Colombia. Este proceso que se realiza con 12 culmos de guadua de $9.00 \mathrm{~m}$, cortados a $1.5 \mathrm{~m}$ para la parte inferior, $4.5 \mathrm{~m}$ para la parte media y $3.00 \mathrm{~m}$ para la parte superior, de estas secciones se seleccionan las probetas para la realización de los ensayos de contenido de humedad, densidad, compresión paralela a la fibra, compresión perpendicular a la fibra, corte con nudo y sin nudo, tensión paralela a la fibra y flexión siguiendo los procedimientos indicados en la Norma Técnica Colombiana y el Reglamento colombiano de construcción sismo resistente NSR 2010. Los resultados de los ensayos fueron sometidos a revisión y análisis para documentar los datos correspondientes a los esfuerzos admisibles y determinación del módulo de elasticidad requerido para la construcción de estructuras con Guadua angustifolia Kunth en todo el territorio nacional.
\end{abstract}

\section{Palabras claves:}

Guadua angustifolia Kunth, ensayos físico-mecánicos, contenido de humedad, esfuerzo admisible, módulo de elasticidad, densidad.

\begin{abstract}
The objective of the current research is to determine the physical and mechanical properties of the bamboo variety Guadua angustifolia Kunth from the municipalities of Socorro- Santander, GuaduasCundinamarca, Oiba -Santander and from the department of Tolima, Colombia. To realize the tests, there were required 12 culms from each of the municipalities, these tests were conducted under moisture content, density, bending stress, shear stress parallel to the fiber, tension stress and parallel and perpendicular compression stress to the fibers, following the procedure described in the Technical Standard
\end{abstract}


Comparación de las propiedades físico-mecánicas del bambú Guadua angustifolia Kunth de diferentes municipios de Colombia

of Colombia 5525 and the Seismic resistant Construction Regulations in Colombia NSR 10. The results obtained by the test were analyzed to collect the data corresponding to the allowable stress and elastic modulus required for constructions of Guadua angustifolia Kunth structures in Colombia.

Keywords: Guadua angustifolia Kunth, physical and mechanical tests, moisture content, admissible stress, elastic modulus, density.

\section{INTRODUCCIÓN}

Debido a la creciente explotación de recursos naturales no renovables es necesario revisar nuevas alternativas para revertir o mitigar el impacto sobre el planeta y sus ecosistemas. La explotación de Guadua angustifolia Kunth se revierte a los 3 años,(Rodríguez, 2007) lo que permite una explotación responsable del recurso, que se puede recuperar rápidamente y de paso colaborar con la reforestación de zonas afectadas por la tala de bosques, desecamiento de los ríos y monocultivos.

Los cultivos de guadua se utilizan como plan de reforestación de los ríos afectados por la minería y la ganadería, es tanto el aumento de la siembra de guadua que se ha dinamizado la construcción de vivienda y espacios ecoturísticos con este material. (Moreno, Osorio, \& Trujillo, 2006; Takeuchi \& González, 2007). Por este motivo es necesario saber las características del material con el fin de garantizar su buen comportamiento en la construcción.

Durante el desarrollo de la vivienda rural colombiana, se observa el uso de guadua sobre todo en estructuras de cubierta y usos ornamentales, las técnicas artesanales de construcción continúan vigentes y con la creciente demanda del material para uso estructural, se han venido refinando para su uso en construcciones más complejas. Desde la década de 1970 se inició la investigación de las propiedades físico-mecánicas del material, desarrollando las primeras metodologías para su estudio y regularización de su uso, de este proceso se destaca que la guadua era utilizada mayormente en la construcción de muros de bahareque.

Para poder dinamizar la construcción con Guadua angustifolia Kunth, se debe realizar un análisis profundo de sus características físico-mecánicas referenciando la ubicación de la guadua en una zona determinada. En Colombia la Guadua angustifolia Kunth se encuentra en su mayoría en los departamentos de Antioquia, Cauca, Caldas, Cundinamarca, Huila, Quindío, Risaralda, Tolima y Valle del Cauca. Debido a varios programas de reforestación la guadua ha venido creciendo en los departamentos de Boyacá, Santander y Norte de Santander.

Como consecuencia del aumento en la siembra de Guadua angustifolia Kunth, en estas zonas del país se han comenzado a construir estructuras con este material, haciendo necesario realizar estudios en diferentes regiones, teniendo en cuenta la trazabilidad de su producción, explotación, tratamientos como inmunización, tipos de cortes, uniones, almacenamiento, transporte, rentabilidad, estudio de mercado, entre otros, con el fin de incentivar su uso.

Desde el aspecto técnico se han realizado estudios donde se caracterizan las diferentes variedades (kunth, amplexifolia, macana, castilla, nigra, cebolla), esta investigación se inició seleccionando la especie Guadua angustifolia Kunth, continuando con la línea de investigación del Grupo de Investigación Madera y guadua de la Universidad Nacional de Colombia, acerca de las caracterizaciones de sus propiedades físico-mecánicas en diferentes lugares del país (Ardila Pinilla, 2013; Garzón Aponte, 2016; Narváez Estefan, 2017) , siguiendo la metodología descrita en la NTC 5525 y los procedimientos mencionados en la NSR 10. Cabe aclarar que la Guadua angustifolia Kunth es la variedad que se encuentra normalizada en la NSR-10.

Se realizaron ensayos mecánicos de Flexión, Corte, compresión paralela y perpendicular a la fibra y tensión paralela con el fin de determinar los valores de esfuerzos admisibles de la Guadua angustifolia Kunth de Socorro, Santander. El procedimiento de los ensayos de corte perpendicular a la fibra se menciona en el libro "Validación de 
la Guadua angustifolia como material estructural para diseño, por el método de esfuerzos admisibles" desarrollado por la Universidad Nacional de Colombia. (AIS, 2010; Instituto Colombiano de Normas Técnicas ICONTEC, 2007)

\section{PRELIMINARES}

\subsection{SELECCIÓN DEL MATERIAL:}

La selección de los culmos se realizó con personal capacitado para la explotación correcta de la Guadua angustifolia Kunth siguiendo las indicaciones de la NTC 5525 y de la NSR 10, se tuvieron en cuenta parámetros como la edad del culmo, la cual debe ser entre 3 y 6 años; los culmos deben tener una circunferencia de entre 32 a $36 \mathrm{~cm}$ a una altura de $1.20 \mathrm{~m}$. no deben tener grietas, agujeros ni presencia de hongo rojo en toda su longitud. En la figura $1 \mathrm{se}$ observa el guadual durante el procedimiento de selección del material.

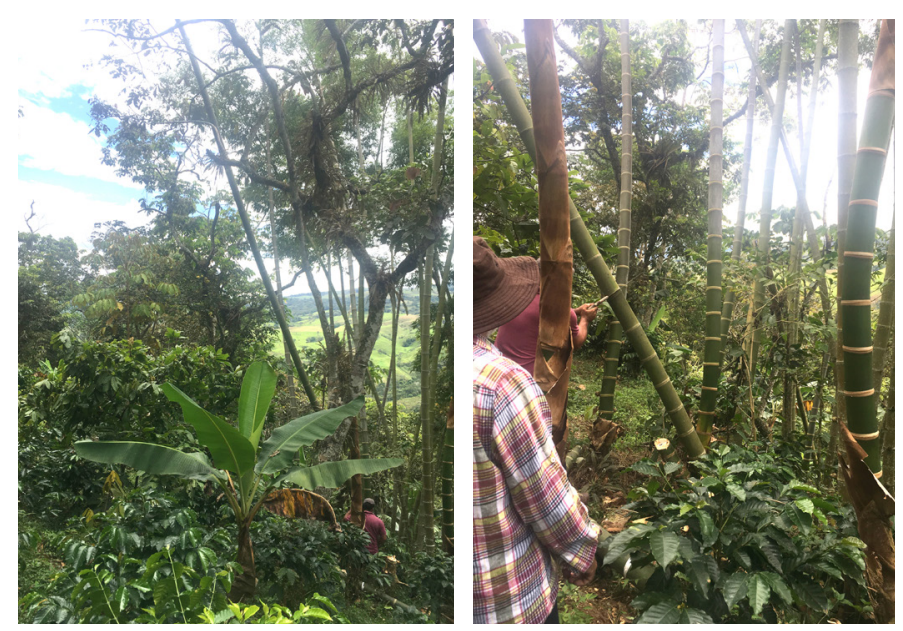

Figura 1. Lugar de suministro de la Guadua angustifolia Kunth.

Una vez seleccionados los 12 culmos se procedió al corte, este se realiza contando a 3 entrenudos desde la base con el fin de permitir que la planta vuelva a brotar. Como se observa en la figura 2.

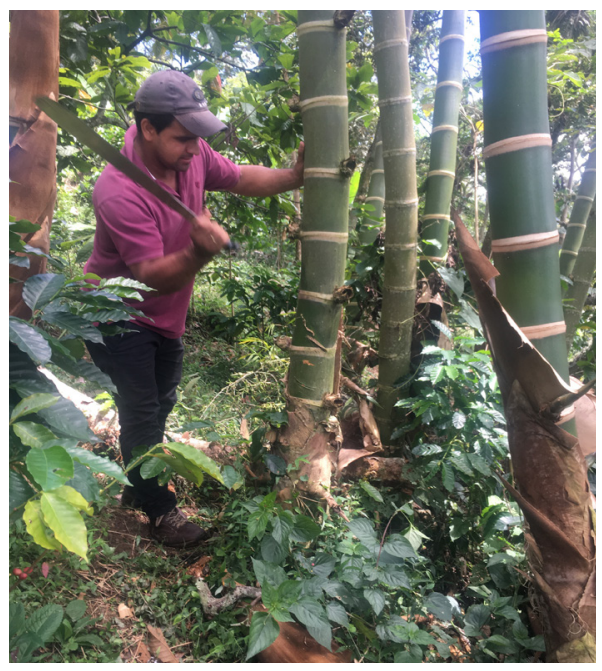

Figura 2. Corte del culmo.

Luego de realizar la tala de la guadua, cada culmo se dividió en tres secciones; inferior de $1.5 \mathrm{~m}$, media de $4.5 \mathrm{~m}$. y superior de $3.5 \mathrm{~m}$; el material cortado se transportó al laboratorio de la Universidad Nacional de Colombia en la ciudad de Bogotá. Las guaduas llegaron al laboratorio 4 días después del corte, es primordial que la guadua para 
Comparación de las propiedades físico-mecánicas del bambú Guadua angustifolia Kunth de diferentes municipios de Colombia

los ensayos sea transportada lo más pronto posible para evitar deterioro en sus condiciones físicas y además de esta manera se reduce el riesgo de ataque de insectos.

\section{CARACTERIZACIÓN FÍSICA Y PREPARACIÓN DE PROBETAS}

\subsection{IDENTIFICACIÓN DE CULMOS Y CORTE DE PROBETAS}

Para la identificación de los culmos se siguieron los siguientes parámetros:

- El material se almacena aislado de la intemperie y evitando su contacto con el suelo

- Se organizan los 12 culmos identificando las diferentes secciones (inferior, media, superior) según la marcación realizada al momento de su corte.

- Se cortan e identificar la cantidad de nudos y entrenudos que tiene cada culmo como se observa en la figura 3.

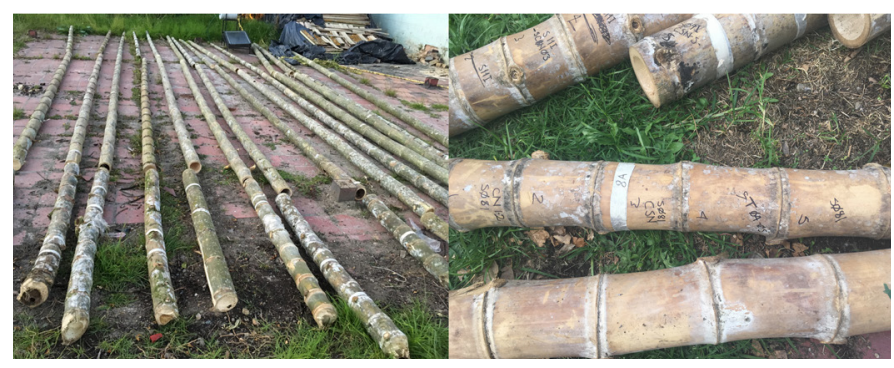

Figura 3. Acopio, marcación de entrenudos.

- Se miden los diámetros de los extremos de cada sección identificando el sentido de crecimiento de la guadua.

- Se realiza la medición de cuatro espesores en los extremos de cada sección.

- Es importante identificar los defectos presentes tales como rajaduras, curvaturas pronunciadas, huecos, etc. Esta información es importante para elegir las probetas.

Esta información obtenida se recolectó en un formato digital. Ver figura 4.

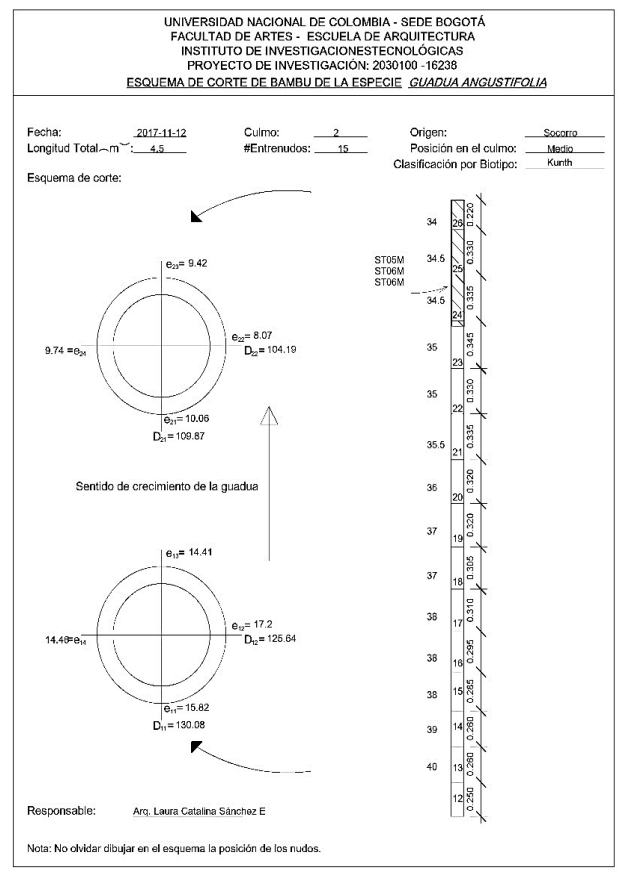

Figura 4. Formato de caracterización. 
Para la enumeración de las probetas se utilizó el sistema mostrado en la figura 5.

\begin{tabular}{|c|c|c|c|c|}
\hline \multicolumn{5}{|c|}{ LUGAR } \\
\hline SOCORRO= $\mathrm{S}$ & GUADUAS= $\mathrm{G}$ & OIBA=O & \multicolumn{2}{c|}{ TOLIMA $=\mathrm{T}$} \\
\hline \multicolumn{5}{|c|}{ ENSAYO } \\
\hline $\begin{array}{c}\text { COMPRESIÓN } \\
\text { PARALELA=C }\end{array}$ & $\begin{array}{c}\text { COMPRESIÓN } \\
\text { ERPENDICULAR=CP }\end{array}$ & $\begin{array}{c}\text { CORTE CON NUDO=V } \\
\text { CORTE SIN NUDO= V }\end{array}$ & $\begin{array}{c}\text { TENSIÓN } \\
\text { PARALELA= T }\end{array}$ & FLEXIÓN = F \\
\hline \multicolumn{5}{|c|}{ SECCIÓN } \\
\hline \multicolumn{7}{|c|}{ MEDIA= M } & SUPERIOR= S \\
\hline
\end{tabular}

Figura 5. Nomenclatura de las probetas.

Luego de definir las probetas y su ubicación se procede a marcar cada probeta en el culmo correspondiente, identificando exactamente la ubicación y el nombre dado a cada probeta siguiendo la nomenclatura indicada anteriormente como se observa en la figura 6.

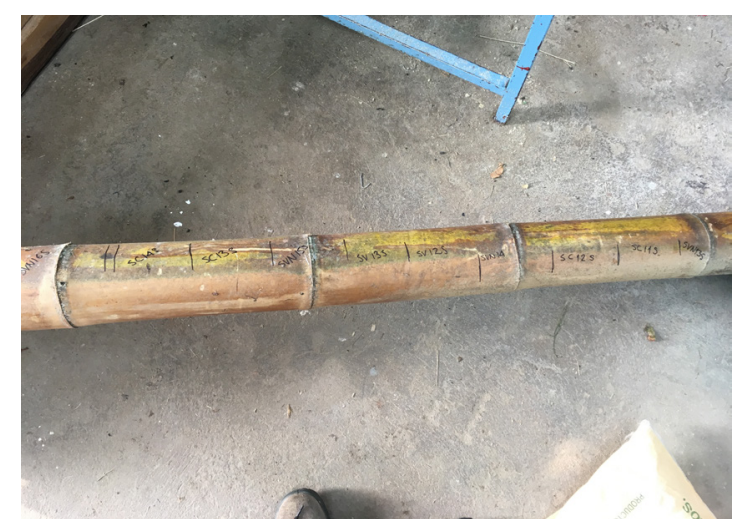

Figura 6. Marcación de las probetas con la nomenclatura.

\subsection{CORTE Y PREPARACIÓN DE LAS PROBETAS}

Luego de realizar la marcación de las probetas se realiza el corte, para este procedimiento se requiere una sierra de disco. Para las probetas de ensayos a tensión paralela se necesita una sierra sinfín debido a su forma curva.

Para los ensayos de compresión y corte paralelo es necesario realizar un proceso de afinado con lija para garantizar un ángulo recto en los extremos respecto a la parte longitudinal.

Luego de todo el proceso de corte y lijado, las probetas se sumergen en agua por un período no menor a 15 días antes de cada ensayo con el fin de saturar el material como se muestra en la figura 7.

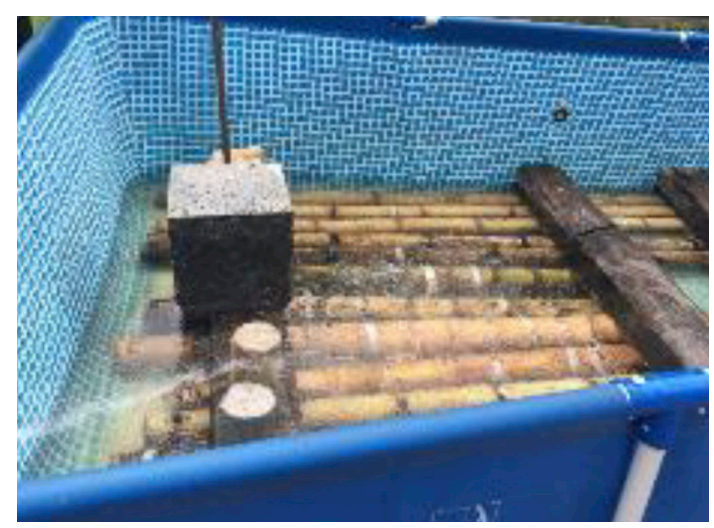

Figura 7. Probetas sumergidas en agua. 


\section{INTRODUCCIÓN A LOS ENSAYOS}

Los ensayos realizados son necesarios para la determinación de los valores de esfuerzo admisible para diferentes solicitaciones, se siguió la metodología descrita en la NTC 5525 "Métodos de ensayo para determinar las propiedades físicas y mecánicas de la Guadua angustifolia Kunth". Para los ensayos de compresión perpendicular a la fibra se utilizó la metodología indicada en el documento "Validación de la Guadua angustifolia como material estructural para diseño, por el método de esfuerzos admisibles" (MADR UNAL, 2010).

\subsection{CRITERIO DE CHAUVENET}

Para cada uno de los ensayos se realizó un análisis estadístico, donde el número de datos se da después de excluir los resultados atípicos usando el Criterio de Chauvenet.

Ecuación para determinar datos atípicos mediante el criterio de Chauvenet.

$$
\frac{x-\dot{\mathrm{x}}}{S}
$$

\subsection{DETERMINACIÓN DEL VALOR CARACTERÍSTICO Y EL ESFUERZO ADMISIBLE}

Luego de realizar el análisis estadístico de cada ensayo, es necesario obtener el valor de los esfuerzos admisibles, determinándolos según los valores característicos de cada solicitación. Para obtener este valor característico debe usarse la ecuación 4.2 usada en la NSR 10 G.12.7-1, esta ecuación se encuentra en la norma ISO 22156:2004

$$
R k=R_{0.05 i}\left(1-\frac{2.7^{\frac{s}{m}}}{\frac{m}{\sqrt{n}}}\right)
$$

En donde:

$R_{k i} \quad=$ es el valor característico en la solicitación i.

$R_{0.05 i}=$ es el percentil 5 de los datos en la solicitación $\mathrm{i}$.

$s \quad=\quad$ es la desviación estándar de los datos de las pruebas de laboratorio.

$m \quad=$ es el promedio de los datos de las pruebas de laboratorio.

$n \quad=$ es el número de datos del ensayo.

$i \quad$ = subíndice que depende del tipo de solicitación (f para flexión, t para tracción paralela a las fibras, $\mathrm{c}$ para compresión paralela a las fibras, $\mathrm{cp}$ para compresión perpendicular a las fibras, $\mathrm{v}$ para cortante paralelo a las fibras)

Luego de hallar el valor característico para cada tipo de ensayo, se determinó el esfuerzo admisible según la ecuación 4.3 usada en el documento MADR-UNAL-2010 y tomada de la Norma ISO 22156:2004 (Bamboo structural design) numeral 7.4.

$$
\sigma_{a d m i}=R_{k i} x G x \frac{D}{S}
$$


En donde:

$\square$ admi $=$ es el esfuerzo admisible.

$R_{k i}=$ es el valor característico para la solicitación i

$G=$ es el coeficiente de modificación para la diferencia entre la calidad del laboratorio y la práctica cuyo valor predeterminado es 0.5 .

$D=$ es el coeficiente de modificación por duración de la carga (1.0 para carga permanente (M), 1.25 para carga permanente más carga temporal $(\mathrm{M}+\mathrm{V})$ y 1.5 para carga permanente más carga temporal más carga de viento $(\mathrm{M}+\mathrm{V}+\mathrm{W}))$.

$S=$ es el factor de seguridad cuyo valor predeterminado es 2.25 .

$i=$ subíndice que depende del tipo de solicitación ( $f$ para flexión, $t$ para tracción paralela a las fibras, c para compresión paralela a las fibras, cppara compresión perpendicular a las fibras, $v$ para cortante paralelo a las fibras).

Con el fin de realizar comparación de resultados, se usó la ecuación 4.4 establecida en la NSR-10 (G.12.7-2), esta ecuación es diferente a la tomada de la norma ISO22156.

$$
F_{i}=\left(\frac{F C}{F_{s} x F D C} F_{k i}\right) c_{m}
$$

$F_{i}=$ es el esfuerzo admisible en la solicitación i

$F_{k i}=$ Valor Característico $17.47 \mathrm{MPa}$

$F C=$ Factor de reducción reducción por diferencias entre las condiciones de los ensayos en el laboratorio y las condiciones reales de aplicación de las cargas en la estructura (1.0 para flexión, 0.5 para tracción, 1.0 para compresión paralela, 1.0 para compresión perpendicular y 0.6 para corte).

$F_{s}=$ es el factor de seguridad (2.0 para flexión, 2.0 para tracción, 1.5 para compresión paralela, 1.8 para compresión perpendicular y 1.8 para corte).

FDC = factor de duración de carga (1.5 para flexión, 1.5 para tracción, 1.2 para compresión paralela, 1.2 para compresión perpendicular y 1.1 para corte).

$\mathrm{C}_{\mathrm{m}}=$ Coeficiente de modificación por $\mathrm{CH}$.

Adicionalmente se tendrán en cuenta la metodología descrita en la NSR-10 correspondientes a la determinación de los esfuerzos admisibles (G.12.7-1) y Módulos de Elasticidad (G.12.7-2).

Tabla 1.

Valores de esfuerzos admisibles. Fuente: NSR-10. (AIS, 2010).

Esfuerzos admisibles $\mathrm{F}_{1}(\mathrm{MP} 3)_{1} \mathrm{CH}=12 \%$

\begin{tabular}{|c|c|c|c|c|}
\hline$\underset{\text { Flexion }}{\mathrm{F}_{\mathrm{h}}}$ & $\underset{\text { Tracción }}{\mathrm{F}_{1}}$ & $\underset{\text { Compresión }}{\mathrm{F}_{\mathrm{t}}}$ & $\underset{\text { Compresion }}{\mathrm{F}_{p+}}$ & $\begin{array}{r}\mathrm{F}_{\mathrm{V}} \\
\text { Corte }\end{array}$ \\
\hline 15 & 18 & 14 & 1.4 & 1.2 \\
\hline
\end{tabular}

$\|$ = ccmpresión paralela sl eje bnglitudingl.

$\perp=$ compresisn perpendicular al eje longitudinal.

"La resatencle a le compresion perpendcular esté ceiculada para entrenudoe relleros con mortaro " de camento. 
Tabla 2.

Valores de módulos de elasticidad Fuente: NSR-10. (AIS, 2010).

Módulos de elasticidad, $\mathrm{E}_{\mathrm{i}}(\mathrm{MPa}), \mathrm{CH}=12 \%$

\begin{tabular}{|c|c|c|}
\hline $\begin{array}{c}\text { Módulo promedio } \\
\mathrm{E}_{0,5}\end{array}$ & $\begin{array}{c}\text { Módulo percentil 5 } \\
\mathrm{E}_{00,5}\end{array}$ & $\begin{array}{c}\text { Módulo minimo } \\
\mathrm{E}_{\text {min }}\end{array}$ \\
\hline 9.500 & 7.500 & 4.000 \\
\hline
\end{tabular}

Tabla 3.

Coeficientes de modificación por contenido de humedad $\left(c_{m}\right)$ Fuente: NSR-10. (AIS, 2010)

\begin{tabular}{|c|c|c|c|c|c|c|c|c|c|}
\hline \multicolumn{2}{|c|}{ Esfuerzos } & $\mathrm{CH} \leq 12 \%$ & $\mathrm{CH}=13 \%$ & $\mathrm{CH}=14 \%$ & $\mathrm{CH}=15 \%$ & $\mathrm{CH}=16 \%$ & $\mathrm{CH}=17 \%$ & $\mathrm{CH}=18 \%$ & $\mathrm{CH} \geq 19 \%$ \\
\hline Flexión & $F_{b}$ & 1.0 & 0.96 & 0.91 & 0.87 & 0.83 & 0.79 & 0.74 & 0.70 \\
\hline Tracción & $F_{t}$ & 1.0 & 0.97 & 0.94 & 0.91 & 0.89 & 0.86 & 0.83 & 0.80 \\
\hline $\begin{array}{c}\text { Compresión } \\
\text { paralela }\end{array}$ & $F_{C}$ & 1.0 & 0.96 & 0.91 & 0.87 & 0.83 & 0.79 & 0.74 & 0.70 \\
\hline $\begin{array}{l}\text { Compresión } \\
\text { perpendicular }\end{array}$ & $F_{p}$ & 1.0 & 0.97 & 0.94 & 0.91 & 0.89 & 0.86 & 0.83 & 0.80 \\
\hline Corta & $F_{y}$ & 1.0 & 0.97 & 0.94 & 0.91 & 0.89 & 0.86 & 0.83 & 0.80 \\
\hline $\begin{array}{l}\text { Modulo de } \\
\text { elasticidad }\end{array}$ & \begin{tabular}{|l|}
$\mathrm{E}_{0,5}$ \\
$\mathrm{E}_{00,5}$ \\
$\mathrm{E}_{\min }$
\end{tabular} & 1.0 & 0.99 & 0.97 & 0.96 & 0.94 & 0.93 & 0.91 & 0.90 \\
\hline
\end{tabular}

\section{ENSAYO DE CONTENIDO DE HUMEDAD}

Para la elaboración de los ensayos de contenido de humedad se siguió la metodología de NTC 5525, el ensayo se elabora calculando la pérdida de masa durante los ciclos de secado de forma progresiva. Se calculó el porcentaje del contenido de humedad de acuerdo a la siguiente ecuación:

Contenido De humedad Fuente: NTC 5525

$$
C H=\frac{m-m_{0}}{m_{0}} \times 100
$$

En donde:

$\mathrm{m}=$ masa de la probeta antes del secado.

mo=masa de la probeta después del secado.

\subsection{COMPARACIÓN DE CONTENIDO DE HUMEDAD (CH)}

La guadua con menor contenido de humedad inicial fue la del municipio de Oiba (19\%) CH, Las guaduas con mayor contenido de humedad fueron las de la Socorro, Santander $(126 \%) \mathrm{CH}$, la guadua de Socorro- Santander incrementó su contenido de humedad de un $42 \%$ a $126 \%$ variando un $84 \%$, fue la guadua que tuvo más variación en su $\mathrm{CH}$. el menor cambio lo tuvo la guadua MADR con un incremento en el $\mathrm{CH}$ de $28 \%$ 


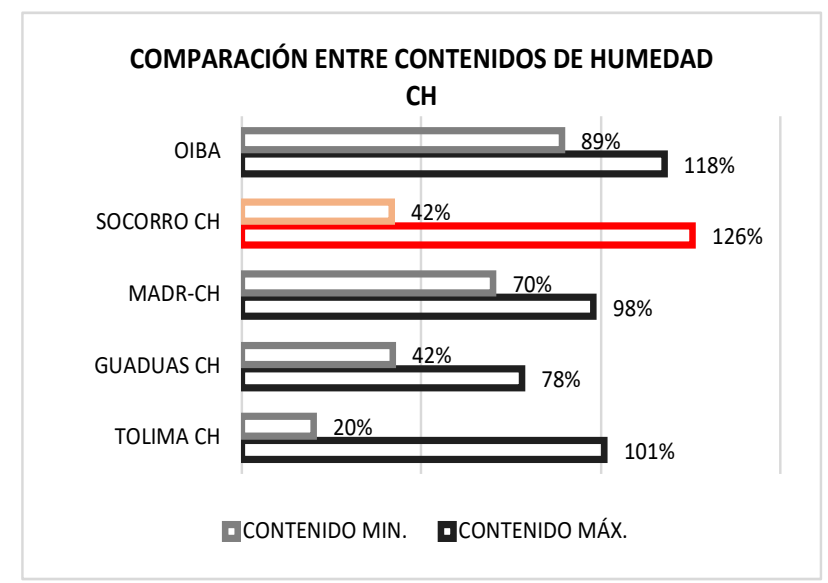

Figura 8. Comparación entre contenidos de humedad $(\mathrm{CH})$. Fuente: Autor.

\section{ENSAYO DE COMPRESIÓN PARALELA A LAS FIBRAS}

Según lo indicado en la NTC 5525 aplicó una carga de $0.01 \mathrm{~mm} / \mathrm{s}$, a probetas con longitud igual al diámetro exterior de la guadua, debe garantizarse la aplicación uniforme de la carga de la probeta, para reducir la fricción se utilizaron placas de acero delgadas en los dos extremos de la probeta como se observa en la figura 9.
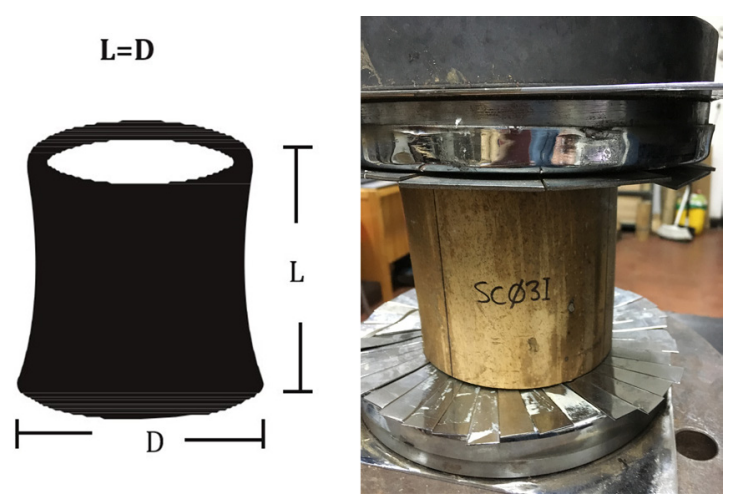

Figura 9. Montaje y dimensiones de la probeta para el ensayo de compresión paralela a las fibras.

Con el fin de hallar el módulo de elasticidad longitudinal (E) a compresión paralela a las fibras, a la mitad de las probetas del ensayo se les instaló una galga extensométrica ubicada en la mitad de su longitud para medir el acortamiento en el sentido longitudinal de las fibras. Los datos fueron recolectados por un adquisidor de datos Kyowa PCD-300 como se observa en la figura 10.

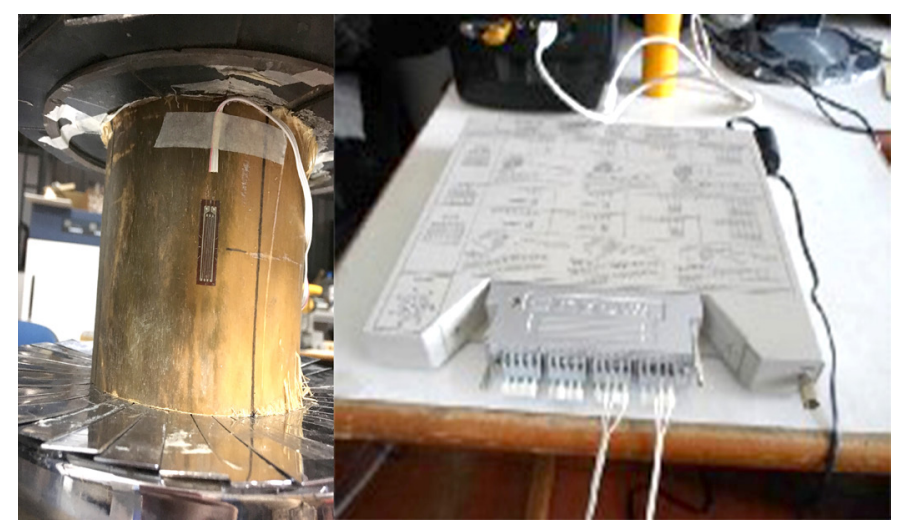

Figura 10. Equipos necesarios para el ensayo de Compresión paralela a las fibras. 
Después de realizado el ensayo, se pudieron observar las fallas típicas de las probetas (figura 6-3), las cuales consisten en la separación de las fibras en los extremos superior e inferior de la probeta. Otro tipo de falla consiste en la aparición de fisuras verticales alrededor de toda la pared externa de la probeta.

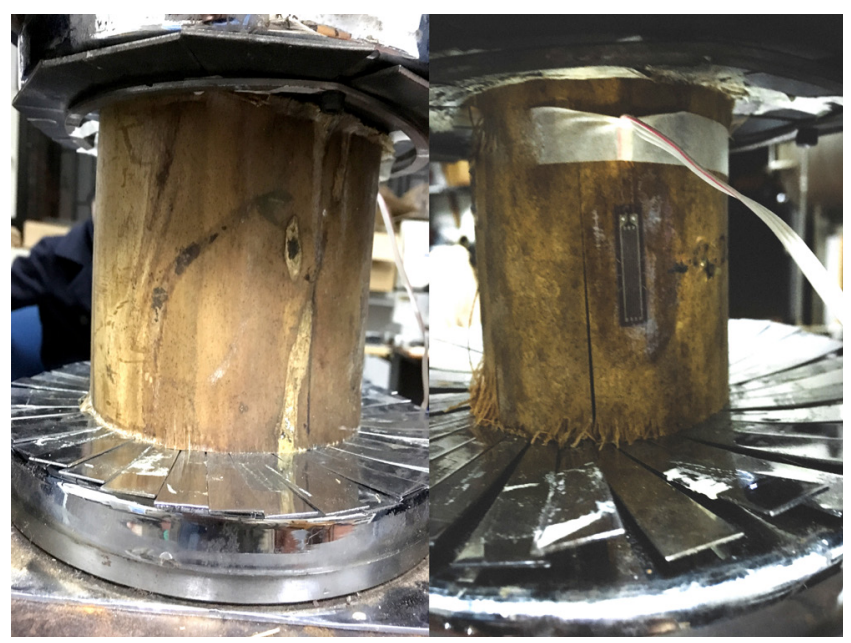

Figura 11. Tipos de fallas típicas en el ensayo de Compresión paralela a las fibras.

\subsection{ESFUERZO ULTIMO A COMPRESIÓN PARALELA A LAS FIBRAS}

Para hallar el esfuerzo último de compresión paralela a las fibras se usó la siguiente ecuación tomada de la NTC 5525.

$$
\sigma_{u l t}=\frac{F_{u l t}}{A}
$$

En donde

$\square_{\text {ult }}=$ Esfuerzo último (MPa)

$F_{u l t}=$ Carga máxima de la probeta $(\mathrm{N})$

$A=$ Área de la sección transversal de la probeta $\left(\mathrm{mm}^{2}\right)$

En comparación con investigaciones anteriores, la Guadua angustifolia Kunth de Socorro Santander sólo presenta un mejor comportamiento en el esfuerzo ultimo a compresión respecto a la Guadua de Oiba, Santander, que tiene un promedio de 22,86 MPa y un comportamiento más cercano a la guadua de Guaduas, Cundinamarca que tiene un promedio de $37,95 \mathrm{MPa}$.

Tabla 4. Comparación de resultados esfuerzo último a compresión paralela.

\begin{tabular}{|c|c|c|c|c|}
\hline DATOS ESTADÍSTICOS & TOLIMA & GUADUAS & OIBA & SOCORRO \\
\hline NÚMERO DE DATOS (N) & 28 & 77 & 77 & 75 \\
\hline PROMEDIO (MPa) & 45,99 & 37,95 & 22,86 & 33,62 \\
\hline DESVIACIÓN ESTÁNDAR (MPa) & 8,04 & 5,59 & 8,21 & 6,95 \\
\hline COEFICIENTE DE VARIACIÓN & $17 \%$ & $15 \%$ & $36 \%$ & $21 \%$ \\
\hline PERCENTIL 5 (MPa) & 34,80 & 29,43 & 6,56 & 23,52 \\
\hline VALOR CARACTERÍSTICO (MPa) & $\mathbf{3 1 , 6 9}$ & $\mathbf{2 , 1 0}$ & $\mathbf{5 , 8 3}$ & $\mathbf{2 2 , 0 2}$ \\
\hline
\end{tabular}




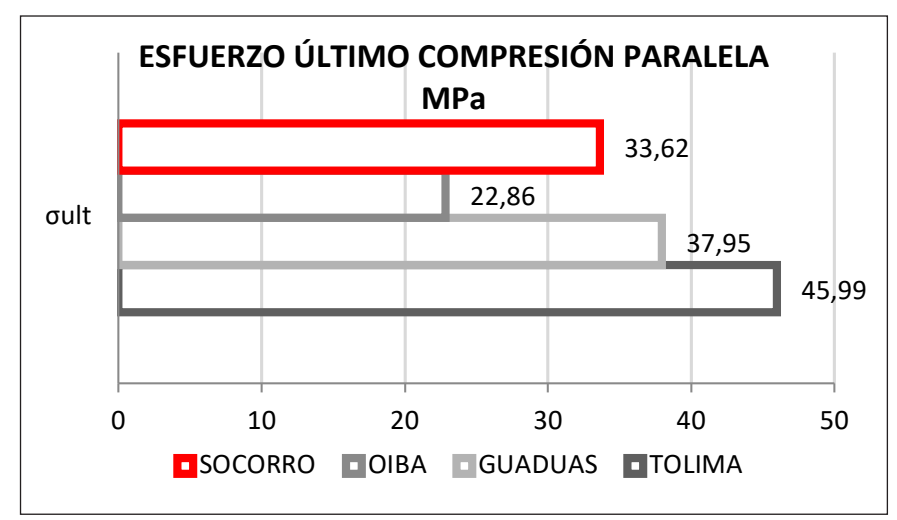

Figura 12. comparación entre regiones esfuerzo último compresión paralela.

\subsection{MÓDULO DE ELASTICIDAD ENSAYO DE COMPRESIÓN PARALELA A LAS FIBRAS}

Posteriormente a la obtención de los datos de desplazamiento $(\mathrm{mm})$ y esfuerzo último (MPa), se calculó la deformación de la probeta y se halló el módulo de elasticidad para el esfuerzo a compresión paralela a las fibras, para esto se realizaron las gráficas de esfuerzo vs deformación para cada probeta y se calculó la pendiente de la recta tangente en el rango elástico del material según el procedimiento indicado en la NTC 5525.

En cuanto al módulo de elasticidad en el ensayo de compresión, para poder comparar los resultados con la NSR10 se realizó el cálculo del módulo de elasticidad mediante las gráficas de esfuerzo vs deformación, los valores obtenidos fueron superiores a lo reportado en la NSR-10 que es de $9500 \mathrm{MPa}$, ver figura 13.

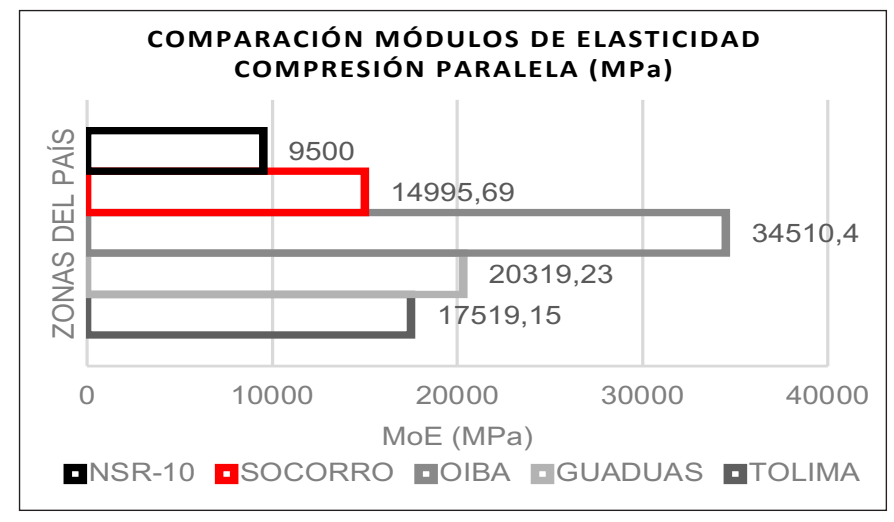

Figura 13. Comparación entre regiones módulo de elasticidad compresión paralela.

\section{ENSAYO DE TENSIÓN PARALELA A LA FIBRA}

Para la elaboración de este ensayo se realizó un montaje según lo descrito en la NTC 5525, en el cual se ejerce una carga de tensión constante a una velocidad de $0.01 \mathrm{~mm} / \mathrm{s}$ por medio de unos sujetadores o mordazas, que garanticen la aplicación de dicha carga a lo largo del eje longitudinal de la probeta y eviten la torsión longitudinal de esta. Las probetas tienen una sección de $10 \mathrm{~cm}, 5 \mathrm{~cm}$ arriba y $5 \mathrm{~cm}$ abajo del nudo, por una sección de 4 a $6 \mathrm{~mm}$, por el espesor de la pared de la guadua, los apoyos miden $25 \mathrm{~mm}$ en su zona de transición y $75 \mathrm{~mm}$ en la zona a ser sujetada por la mordaza como se observa en la figura 14.

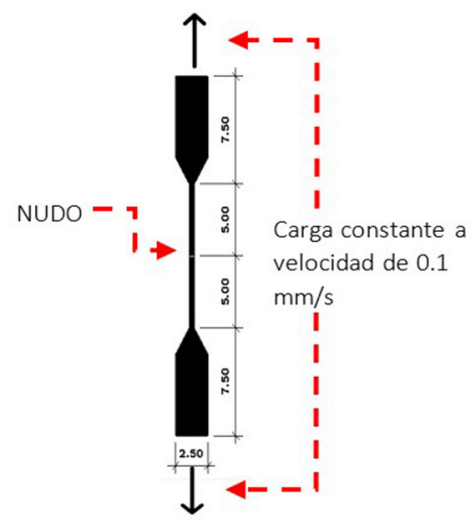

Figura 14. Dimensiones de las probetas para ensayo de Tensión paralela a las fibras. 
Con el fin de determinar el módulo de elasticidad a tensión paralela de las fibras de la Guadua angustifolia Kunth, se instrumentó cada probeta con un extensómetro mecánico (figura 15). El extensómetro, marca Epsilon, se calibró para posicionarlo centrado en la sección de nudo de la probeta, este equipo se conectó al adquisidor de datosPCD-300 marca Kyowa.

La falla típica se produce causando una rotura en el centro de la probeta. La falla se manifiesta mediante el desprendimiento de las fibras en sentido diagonal hasta llegar a la otra cara. Esta falla se repite durante la totalidad de las probetas como se observa en la figura 15.

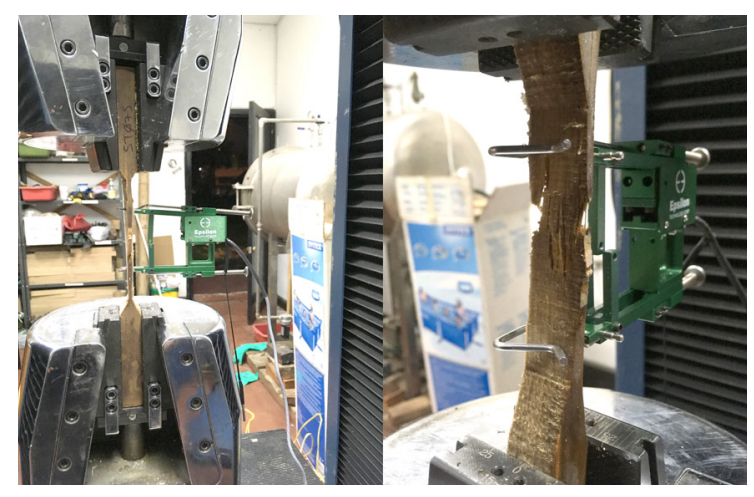

Figura 15. Tipos de fallas típicas en el ensayo de Compresión paralela a las fibras, montaje de extensómetro

\subsection{ESFUERZO ULTIMO A TENSIÓN PARALELA A LAS FIBRAS}

Para determinar el esfuerzo último a los ensayos de tensión paralela a las fibras, se toma como base la ecuación 8.1 extraída de la norma NTC-5525:

$$
\sigma_{u c}=\frac{F_{u l t}}{A}
$$

En donde:

$\square_{\text {ult }}=$ Esfuerzo último

$F_{u l t}=$ carga máxima soportada por la probeta

$A=$ área de la sección transversal de la sección del ensayo.

En comparación del esfuerzo último a tensión, se observó que el valor característico de Tolima es el más alto (56,58 $\mathrm{MPa})$, Socorro, Santander reportó el valor más bajo (22,73 MPa). Debido a que el contenido de humedad de las probetas a tensión paralela a las fibras de Socorro, Santander era mayor que el del resto de las investigaciones $(125.54 \% \mathrm{CH})$. Se puede deducir que afectó el comportamiento de los ensayos, derivando en una resistencia menor al esfuerzo último. Ver figura 16

Tabla 5.

Comparación de resultados esfuerzo último a tensión paralela.

\begin{tabular}{|c|c|c|c|c|}
\hline NÚMERO DE DATOS (N) & TOLIMA & GUADUAS & OIBA & SOCORRO \\
\hline PROMEDIO (MPa) & 56.58 & 50,61 & 56.32 & 42,53 \\
\hline DESVIACIÓN ESTÁNDAR (MPa) & 15.55 & 10,84 & 19.53 & 13,33 \\
\hline COEFICIENTE DE VARIACIÓN & $27.48 \%$ & $21 \%$ & $35 \%$ & $31 \%$ \\
\hline PERCENTIL 5 (MPa) & 34.84 & 31,33 & 27 & 25,23 \\
\hline VALOR CARACTERÍSTICO (MPa) & 30.53 & 29,12 & 23.96 & 22,73 \\
\hline
\end{tabular}




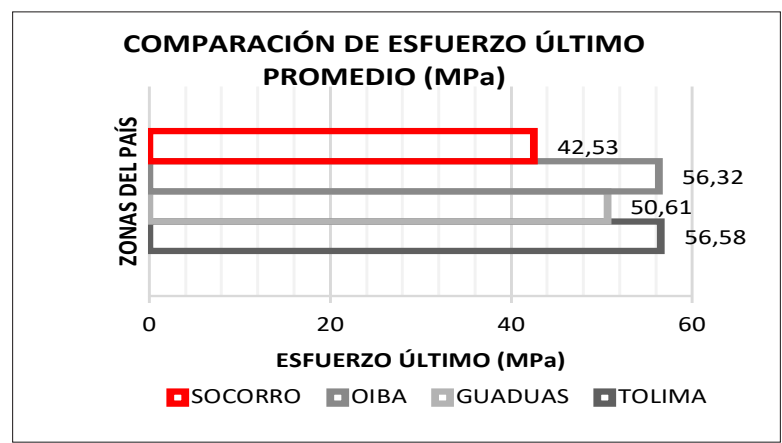

Figura 16. Comparación entre regiones esfuerzo último tensión paralela.

\subsection{MÓDULO DE ELASTICIDAD ENSAYO DE TENSIÓN PARALELA A LAS FIBRAS}

En el ensayo de tensión paralela a las fibras, el mayor valor reportado fue el de Guaduas, Cundinamarca (19486,27 $\mathrm{MPa})$, el menor valor fue el de Oiba, Santander (9425,19 MPa. Todos los valores sobrepasaron al valor reportado en la NSR-10 (9500MPa). Ver figura 17.

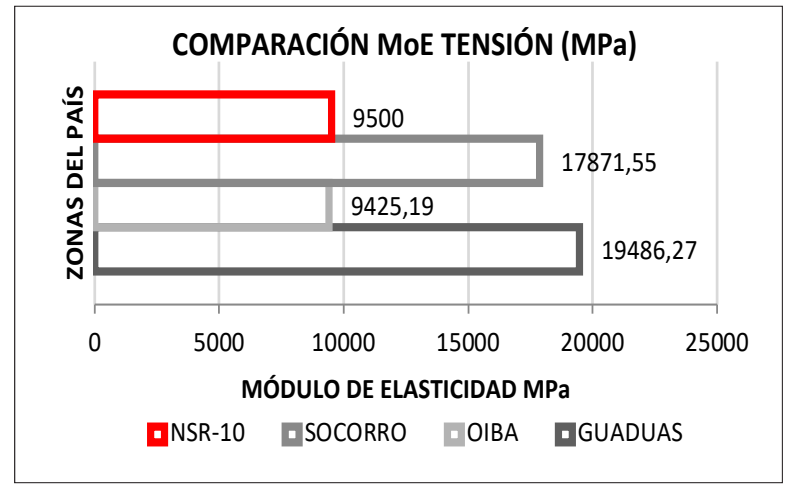

Figura 17. Comparación entre regiones módulo de elasticidad tensión paralela.

\section{ENSAYO DE COMPRESIÓN PERPENDICULAR A LAS FIBRAS}

Para realizar este ensayo se utilizaron probetas de $17 \mathrm{~cm}$ de altura y sin nudo, estas se ubicaron en el centro del plato de carga hasta una posición de reposo natural y se les aplicó una carga pequeña para acomodar la probeta, finalmente se les aplicó una carga constante a una velocidad de $0.01 \mathrm{~mm} / \mathrm{s}$ en el extremo superior como se observa en la figura 8-1, en las fallas típicas de las probetas, se generan fisuras en el sentido longitudinal, al interior en la parte superior e inferior y al exterior en las laterales.
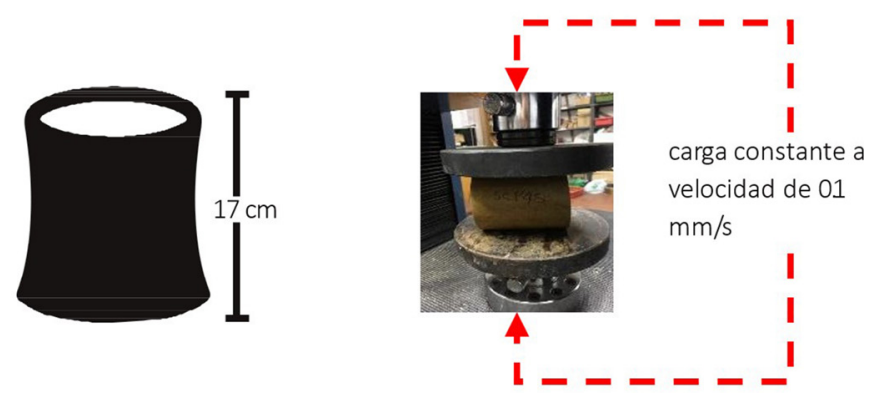

Figura 18. Montaje y dimensiones de la probeta para el ensayo de compresión perpendicular a las fibras.

El esfuerzo último a compresión perpendicular a las fibras $\left(\square_{\text {ucp }}\right)$ se determinó usando la ecuación 8-1 tomada de MADR-UNAL, 2010. 
Comparación de las propiedades físico-mecánicas del bambú Guadua angustifolia Kunth de diferentes municipios de Colombia

$$
\sigma_{u c p}=\frac{3 x D_{\operatorname{exF}}}{2 x L x t^{2}}
$$

En donde:

$D_{e}=$ es el diámetro externo promedio de la probeta. $(\mathrm{mm})$

$F=$ es la carga máxima aplicada (MPa)

$L=$ es la longitud promedio $(\mathrm{mm})$.

$t=$ el espesor de pared promedio $(\mathrm{mm})$.

\subsection{ESFUERZO ULTIMO A COMPRESIÓN PERPENDICULAR}

Para el esfuerzo ultimo a compresión perpendicular, los resultados obtenidos en la comparación arrojaron que la Guadua angustifolia Kunth procedente del municipio de Socorro tiene mejor comportamiento que la guadua de Oiba y Tolima, registrando un esfuerzo último promedio de 7.79 MPa similar al de Guaduas7.81 MPa, zona que registró el mayor valor como se observa en la figura 19.

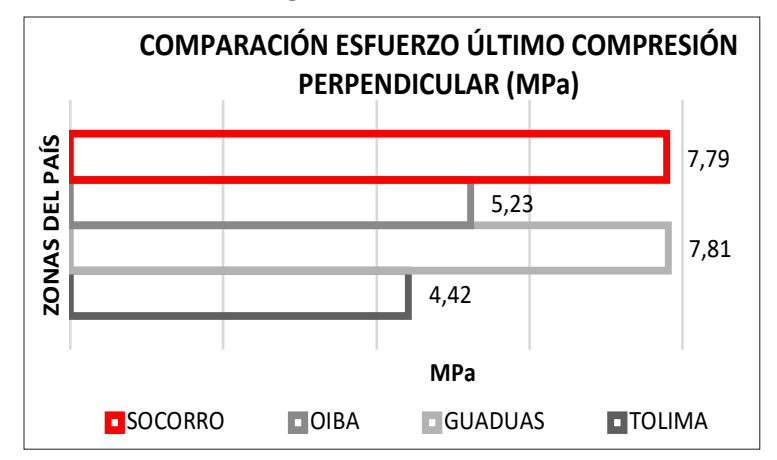

Figura 19. Comparación entre regiones esfuerzo último compresión perpendicular.

\section{ENSAYO DE CORTE PARALELO A LAS FIBRAS}

Para la elaboración de este ensayo se realizó el montaje siguiendo las indicaciones dadas en la NTC 5525, en el montaje se aplicó una carga constante a una velocidad de $0.01 \mathrm{~mm} / \mathrm{s}$. El montaje consistía en la ubicación de las probetas de igual diámetro y longitud, la mitad con nudo y la mitad sin nudo, ubicadas entre 2 placas con perforaciones opuestas mediante las cuales la probeta debía recibir la carga con la finalidad de producir la falla por corte, como se observa en la figura 20.

La falla de estas probetas se manifestó mediante fisuras verticales en los lugares donde estuvo apoyado el elemento. Ver figura 20.
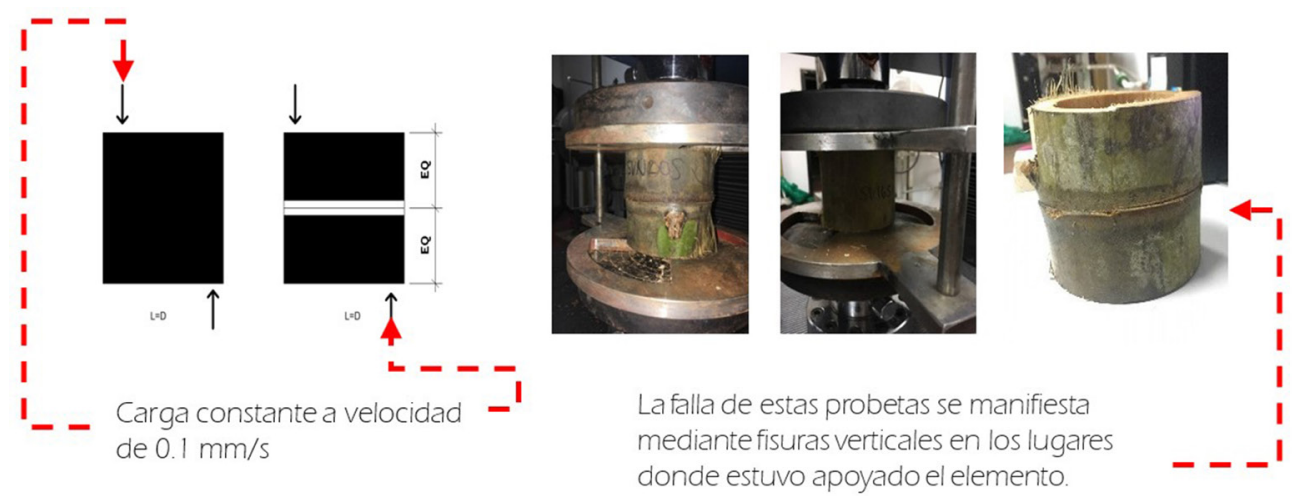

Figura 20. Montaje y dimensiones de la probeta para el ensayo de corte paralelo a las fibras. 
Para calcular el esfuerzo último a corte paralelo a las fibras se utiliza la siguiente ecuación tomada de la NTC 5525:

$$
\sigma_{u v}=\frac{F_{u l t}}{\sum(t x L)}
$$

En donde:

$F_{u t t}=$ es la carga máxima aplicada. (MPa)

$t=$ es el espesor de pared en cada una de las cuatro áreas de corte. $(\mathrm{mm})$

$L=$ es la altura de la probeta en cada una de las cuatro áreas de corte. $(\mathrm{mm})$

\subsection{ESFUERZO ULTIMO CORTE PARALELO A LAS FIBRAS}

En la comparación de resultados se observó que el mayor valor para el esfuerzo último lo obtuvo Guaduas, Cundinamarca (5.16 MPa), el valor de Oiba, Santander fue el más bajo (3,95 MPa). Ver figura 21.

Tabla 6.

Comparación de resultados esfuerzo último corte paralelo.

\begin{tabular}{|c|c|c|c|c|}
\hline $\begin{array}{c}\text { DATOS ESTADÍSTICOS } \\
\text { NÚMERO DE DATOS (N) }\end{array}$ & $\begin{array}{c}\text { TOLIMA } \\
64\end{array}$ & $\begin{array}{c}\text { GUADUAS } \\
76\end{array}$ & $\begin{array}{c}\text { OIBA } \\
75\end{array}$ & $\begin{array}{c}\text { SOCORRO } \\
70\end{array}$ \\
\hline PROMEDIO (MPa) & 4.46 & 7.57 & 3.95 & 7,82 \\
\hline DESVIACIÓN ESTÁNDAR (MPa) & 1.13 & 1.38 & 1.15 & 1,37 \\
\hline COEFICIENTE DE VARIACIÓN & $25.31 \%$ & $18 \%$ & $29 \%$ & $18 \%$ \\
\hline PERCENTIL 5 (MPa) & 2.69 & 5.47 & 2.06 & 5,49 \\
\hline VALOR CARACTERÍSTICO (MPa) & 2.46 & 5.16 & 1.87 & 5.18 \\
\hline
\end{tabular}

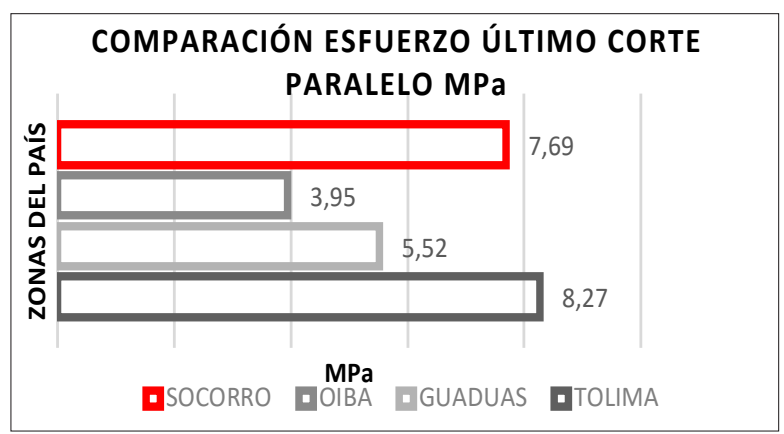

Figura 21. Comparación entre regiones esfuerzo último corte paralelo.

\section{ENSAYO DE FLEXIÓN}

Se ensayaron 12 probetas ante esfuerzo de flexión, las probetas fueron tomadas de la sección media de cada culmo, ya que por requerimiento de la NTC 5525 la longitud de la probeta debe ser 30 veces el diámetro de esta, lo que usualmente sobrepasa los $3 \mathrm{~m}$. de la sección superior y los $1.5 \mathrm{~m}$. de la parte inferior. Para estos ensayos se requiere un montaje en el cual se debe aplicar una carga constante a velocidad de $0.5 \mathrm{~mm} / \mathrm{s}$ en los tercios de la luz libre entre los dos apoyos. Tanto la carga como las reacciones se aplican en los nudos del culmo como se observa en la figura 22.

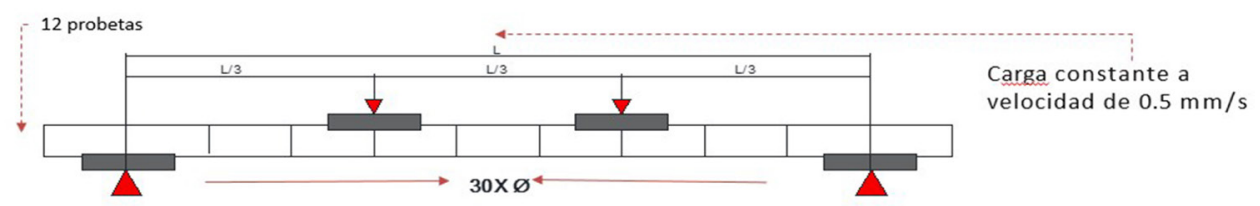

Figura 22. Montaje de la probeta para el ensayo de flexión. 
Para todas las probetas se realizaron los ensayos usando un equipo LVDT en el centro de la luz del elemento para realizar la medición de la deflexión ( $\square$ y luego el cálculo del módulo de elasticidad (E) a flexión.

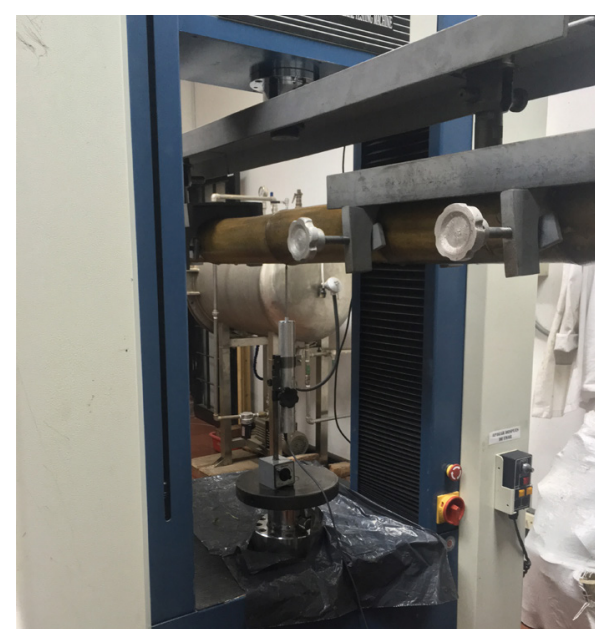

Figura 23. Equipo LVDT.

La falla de la probeta, donde ocurre aplastamiento del elemento, además de rotura del nudo y rajaduras en dirección a las fibras.

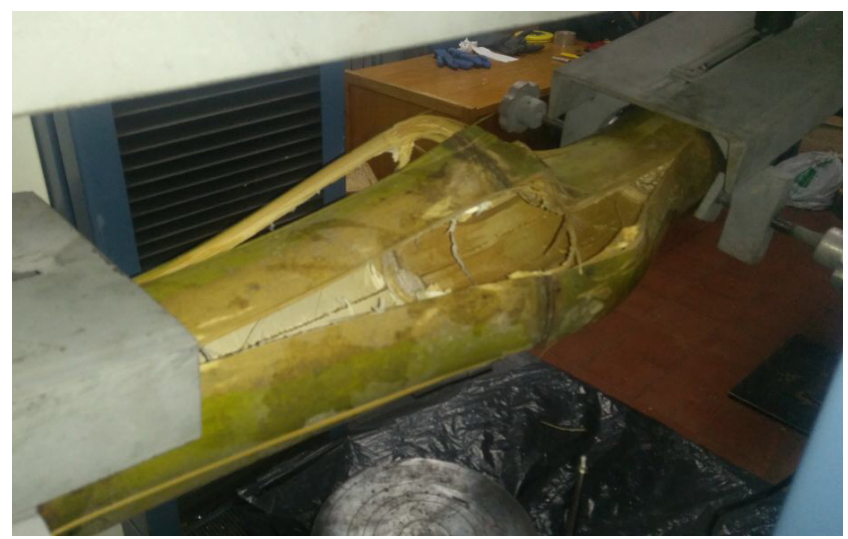

Figura 24. Falla típica del ensayo de flexión.

El momento de inercia (IB) se calculó usando la siguiente ecuación NTC 5525.

$$
I_{B}=\frac{\pi}{64}\left(D^{4}-(D-2 t)^{4}\right)
$$

En donde:

$\mathrm{IB}=$ Momento de inercia

D = Diámetro externo promedio.

$\mathrm{T}=$ Espesor de pared promedio. 


\subsection{ESFUERZO ULTIMO A FLEXIÓN}

El esfuerzo último a flexión ( $\square$ uf) se determinó usando la ecuación 10.2 tomada de la NTC 5525, que está en función del momento de inercia IB de la ecuación 10.1

En donde:

$$
\sigma_{u f}=F x L x \frac{D / 2}{6} \times \frac{1}{I B}
$$

$\square$ uf= Esfuerzo último del ensayo a flexión en MPa

$\mathrm{F}=$ Carga máxima aplicada.

$L=$ Luz libre.

$\mathrm{D}=$ Diámetro externo.

Para el esfuerzo a flexión, en comparación con los resultados obtenidos en investigaciones previas para otras regiones del país se observa que la Guadua angustifolia Kunth procedente del municipio de Socorro tiene un esfuerzo último promedio de 68.39MPa, inferior al valor de 72,82 MPa del municipio de Guaduas, mientras que es mucho mayor al de Oiba que obtuvo un valor de $39 \mathrm{MPa}$.

Tabla 7.

Comparación de resultados esfuerzo último a flexión.

\begin{tabular}{|c|c|c|c|c|}
\hline \multicolumn{5}{|c|}{ ESFUERZO ÚLTIMO } \\
\hline & TOLIMA & $\begin{array}{c}\text { GUADUAS } \\
\text { CUNDINAMARCA }\end{array}$ & $\begin{array}{l}\text { OIBA } \\
\text { SANTANDER }\end{array}$ & $\begin{array}{l}\text { SOCORRO } \\
\text { SANTANDER }\end{array}$ \\
\hline $\begin{array}{l}\text { DATOS } \\
\text { ESTADÍSTICOS }\end{array}$ & $\begin{array}{l}\text { SECCIÓN } \\
\text { MEDIA }\end{array}$ & $\begin{array}{c}\text { SECCIÓN } \\
\text { MEDIA }\end{array}$ & $\begin{array}{l}\text { SECCIÓN } \\
\text { MEDIA }\end{array}$ & $\begin{array}{l}\text { SECCIÓN } \\
\text { MEDIA }\end{array}$ \\
\hline $\begin{array}{l}\text { NÚMERO DE } \\
\text { DATOS (N) }\end{array}$ & 12 & 12 & 11 & 12 \\
\hline PROMEDIO (MPa) & 38.88 & 72.82 & 39.81 & 68,39 \\
\hline $\begin{array}{l}\text { DESVIACIÓN } \\
\text { ESTÁNDAR (MPa) }\end{array}$ & 4.85 & 9.45 & 10.72 & 29,73 \\
\hline $\begin{array}{l}\text { COEFICIENTE DE } \\
\text { VARIACIÓN }\end{array}$ & 12.47 & $13 \%$ & $27 \%$ & $43 \%$ \\
\hline $\begin{array}{l}\text { PERCENTIL } 5 \\
\text { (MPa) }\end{array}$ & 33.9 & 60.42 & 28.18 & 27,63 \\
\hline $\begin{array}{l}\text { VALOR } \\
\text { CARACTERÍSTICO } \\
\text { (MPa) }\end{array}$ & 30.60 & 54.31 & 22 & 18.26 \\
\hline
\end{tabular}

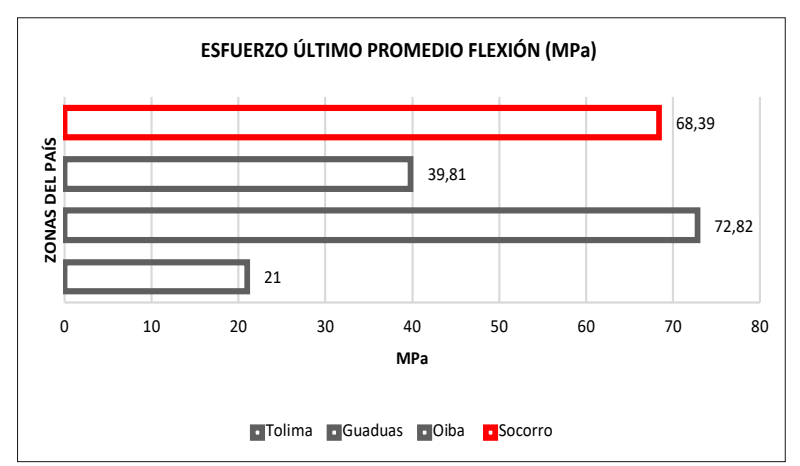

Figura 25. Comparación entre regiones esfuerzo último flexión.

\subsection{MÓDULO DE ELASTICIDAD ENSAYO DE FLEXIÓN}

El módulo de elasticidad (E) a flexión se determinó usando la siguiente ecuación tomada de la NTC 5525.

$$
E=\frac{23 x F x L^{3}}{1296 x \delta \times I_{B}}
$$


Comparación de las propiedades físico-mecánicas del bambú Guadua angustifolia Kunth de diferentes municipios de Colombia

En donde:

$\mathrm{E}=$ Módulo de elasticidad

$\mathrm{F}=$ es la carga máxima aplicada.

$L=$ es la luz libre.

$\mathrm{IB}=$ es el momento de inercia.

$\square=$ es la deflexión en el punto medio de la luz.

En la comparación de los resultados obtenidos por las investigaciones para el módulo de elasticidad calculado en el ensayo de flexión, se observó que la Guadua angustifolia Kunth procedente del Tolima obtuvo el valor más alto (14933 MPa), Los resultados son bastante cercanos entre sí, todos los valores sobrepasan al valor otorgado por la NSR 10 que es de 90500 MPa como se observa en la figura 26.

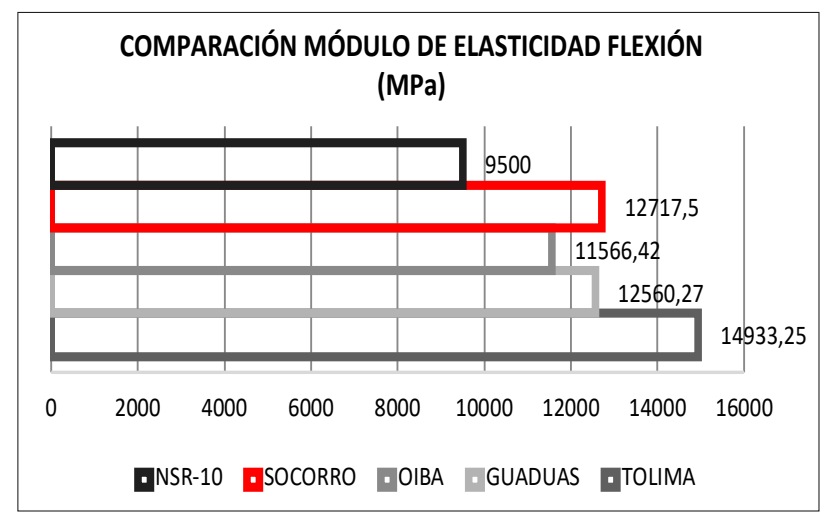

Figura 26. Comparación entre regiones módulo de elasticidad flexión.

\section{ENSAYO DE DENSIDAD}

Para la determinación de la densidad básica de la Guadua angustifolia Kunth, se siguieron los lineamientos establecidos en la NTC 5525, basada en el principio de la determinación de la masa mediante el pesaje y del volumen a través de la medición de sus dimensiones, para el cálculo de la masa por unidad de volumen de Guadua angustifolia Kunth.

$$
\rho=(m / V) \times 10^{6}
$$

En donde:

$\square=$ es densidad, en $\mathrm{Kg} / \mathrm{m} 3$.

$\mathrm{m}=$ es la masa de la probeta seca en horno, en $\mathrm{g}$.

$\mathrm{V}=$ es el volumen húmedo (verde) de la probeta en $\mathrm{mm} 3$

Para la elaboración del ensayo de densidad se utilizaron los siguientes instrumentos como se observa en la figura 27.

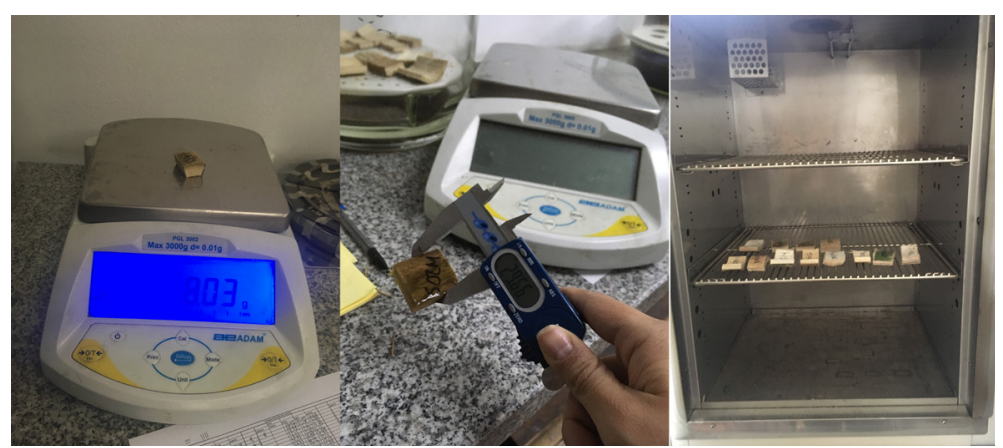

Figura 27 Equipos utilizados para el ensayo de densidad. 
Tabla 8 Resultados ensayo de densidad.

\begin{tabular}{|c|c|c|c|c|}
\hline DATOS & SECCIÓN & SECCIÓN & SECCIÓN & TOTAL \\
ESTADÍSTICOS & INFERIOR & MEDIA & SUPERIOR & TOT \\
\hline NÚMERO DE DATOS (N) & 12 & 12 & 12 & 35 \\
PROMEDIO (Kg/m $\left.{ }^{3}\right)$ & 547,95 & 522,58 & 551,98 & 545,9 \\
& & & & 8 \\
DESVIACIÓN ESTÁNDAR (Kg/m & 27,79 & 31,02 & 15,32 & 40,24 \\
COEFICIE NTE DE VAR IACIÓ N & $5 \%$ & $6 \%$ & $3 \%$ & $7 \%$ \\
\hline
\end{tabular}

\subsection{COMPARACIÓN DE ENSAYOS DE DENSIDAD}

En comparación con los valores de densidad obtenidos para las zonas de Guaduas, Cundinamarca (620.47 kg/ $\mathrm{m} 3)$ y Oiba Santander, $(531.26 \mathrm{~kg} / \mathrm{m} 3)$ se puede observar que la densidad entre Oiba y Socorro es similar, esto puede ser por la cercanía entre la localización de los municipios, factores como la temperatura y la altitud pueden influir en la guadua proveniente de estas zonas como se observa en la figura 28.

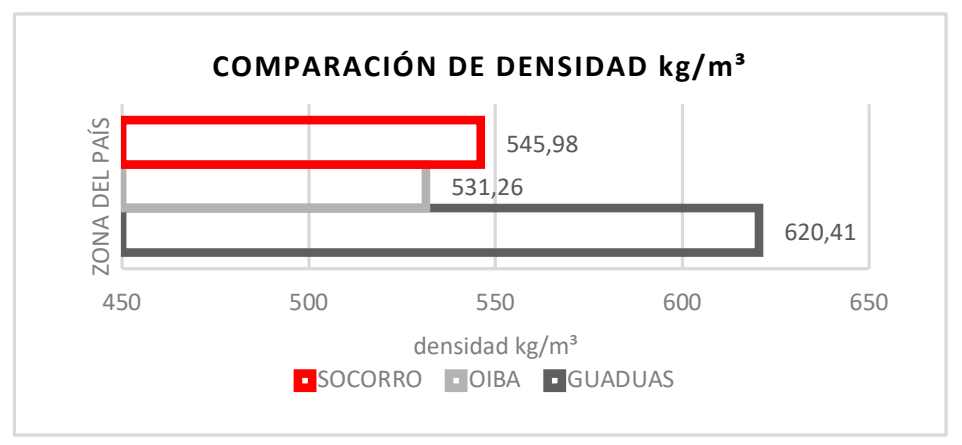

Figura 28. Comparación entre regiones densidad.

\section{DISCUSIÓN}

Durante la caracterización de la Guadua angustifolia Kunth de Socorro, Santander, se encontró que en la NSR 10 hay un apartado donde se indica que los ensayos realizados para el Título $G$ se realizaron bajo un contenido de humedad de $12 \% \mathrm{CH}$, con el fin de poder comparar los resultados obtenidos en los ensayos, la Norma contiene unos coeficientes de modificación por $\mathrm{CH}$, que deben aplicarse a los resultados de esfuerzo admisible y módulo de elasticidad, los valores de estos coeficientes se observan en la tabla 9. Por lo anterior es importante tener en cuenta los coeficientes de modificación por contenido de humedad par próximas caracterizaciones, este paso es muy importante para hacer un comparativo más acertado respecto a los valores especificados en la NSR-10. (Sánchez Escobar, 2018)

Tabla 9. Coeficientes de modificación por CH para esfuerzo admisible. Fuente NSR 10

\begin{tabular}{|c|c|c|c|c|c|c|c|c|c|}
\hline \multicolumn{2}{|c|}{ Esfuerzos } & $\mathrm{CH} \leq 12 \%$ & $\mathrm{CH}=13 \%$ & $\mathrm{CH}=14 \%$ & $\mathrm{CH}=15 \%$ & $\mathrm{CH}=16 \%$ & $\mathrm{CH}=17 \%$ & $\mathrm{CH}=18 \%$ & $\mathrm{CH} \geq 19 \%$ \\
\hline Flexión & $F_{b}$ & 1.0 & 0.96 & 0.91 & 0.87 & 0.83 & 0.79 & 0.74 & 0.70 \\
\hline Tracción & $F_{t}$ & 1.0 & 0.97 & 0.94 & 0.91 & 0.89 & 0.86 & 0.83 & 0.80 \\
\hline $\begin{array}{c}\text { Compresión } \\
\text { paralela }\end{array}$ & $\mathrm{F}_{\mathrm{C}}$ & 1.0 & 0.96 & 0.91 & 0.87 & 0.83 & 0.79 & 0.74 & 0.70 \\
\hline $\begin{array}{l}\text { Compresión } \\
\text { perpendicular }\end{array}$ & $F_{p}$ & 1.0 & 0.97 & 0.94 & 0.91 & 0.89 & 0.86 & 0.83 & 0.80 \\
\hline Corta & $F_{y}$ & 1.0 & 0.97 & 0.94 & 0.91 & 0.89 & 0.86 & 0.83 & 0.80 \\
\hline $\begin{array}{l}\text { Modulo de } \\
\text { elasticidad }\end{array}$ & \begin{tabular}{|l}
$E_{0,5}$ \\
$E_{00,5}$ \\
$E_{\text {min }}$ \\
\end{tabular} & 1.0 & 0.99 & 0.97 & 0.96 & 0.94 & 0.93 & 0.91 & 0.90 \\
\hline
\end{tabular}


Comparación de las propiedades físico-mecánicas del bambú Guadua angustifolia Kunth de diferentes municipios de Colombia

Se recomienda revisar los valores especificados en la NSR-10 en cuanto a esfuerzos admisibles, ya que en los resultados de los ensayos casi ningún valor llega a los exigidos por la norma como se observa en la figura 29.

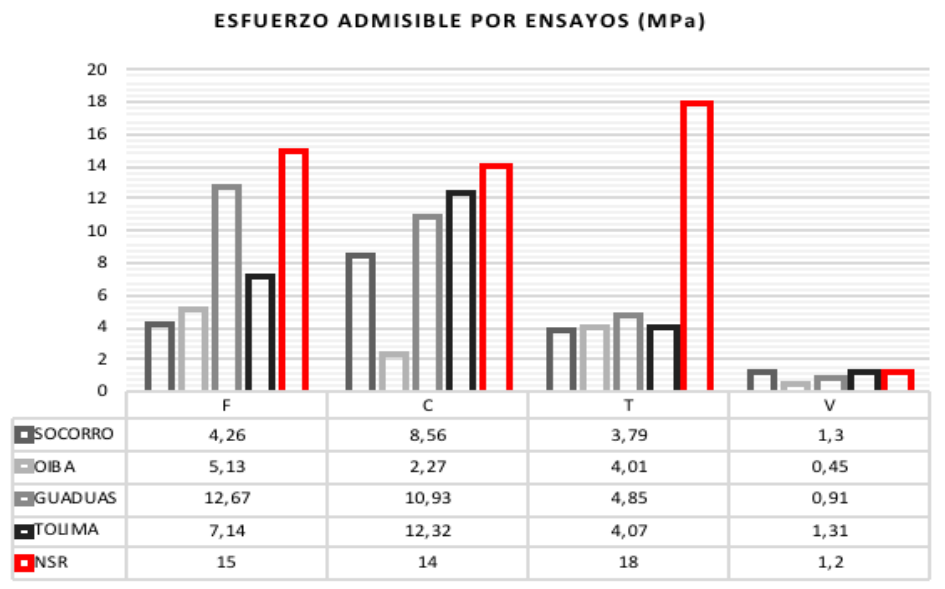

Figura 29. Resultados de Esfuerzos admisibles por ensayos y lugares.

Se debe plantear tanto un tiempo máximo de inmersión en agua de las probetas, así como existe un tiempo mínimo (dos semanas), ya que las probetas al ser de un material vegetal pueden descomponerse afectando la elaboración de probetas y ensayos.

Debido a los resultados heterogéneos que se obtuvieron en las investigaciones, es importante incluir en la NSR-10 los valores de esfuerzo admisible por departamentos y municipios, de esta forma se puede hacer una comparación más específica y con valores reales de las propiedades físico-mecánicas por cada zona del país

\section{CONCLUSIONES}

\subsection{CONTENIDO DE HUMEDAD}

Se halló el contenido de humedad para cada probeta y se clasificó por ensayos, teniendo en cuenta que varía la geometría de la probeta en cada procedimiento. El Contenido de humedad $\mathrm{CH}$, para los culmos obtenidos del Socorro Santander, varía entre las probetas con el menor contenido de humedad (flexión $41.75 \% \mathrm{CH}$ ) y las de tensión paralela tuvieron el mayor contenido de humedad $(126.78 \% \mathrm{CH})$.

Comparando con los estudios realizados a la Guadua angustifolia Kunth de las regiones del Socorro Santander, MADR, Guaduas Cundinamarca y Tolima, la Guadua angustifolia Kunth del Socorro tiene el mayor contenido de humedad $126.78 \%$, mientras que la de Guaduas tiene el menor contenido de humedad $41.43 \%$ como se observa en la figura 30 .

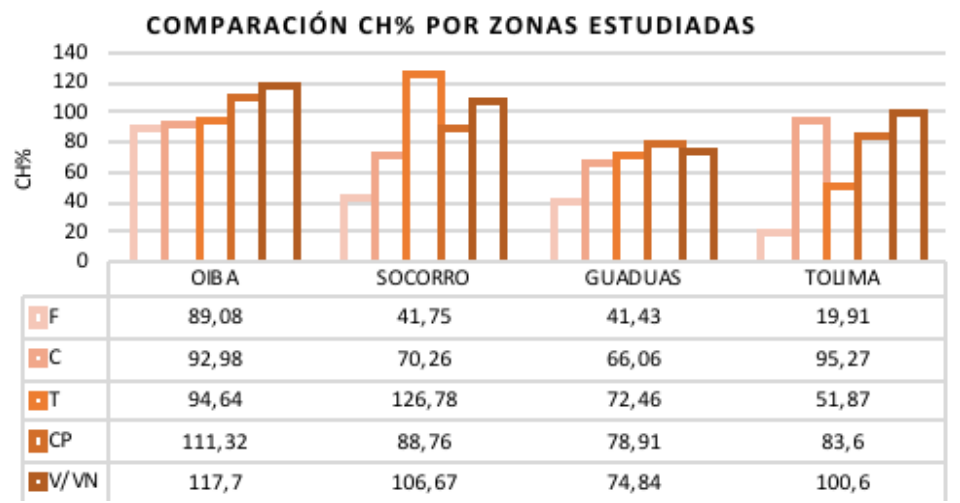

Figura 30. Comparación porcentaje mínimo y máximo de contenido de humedad entre regiones. 


\subsection{ENSAYO A FLEXIÓN}

En comparación con los esfuerzos admisibles de Tolima, Guaduas-Cundinamarca y Oiba-Santander, Socorro Santander tiene el menor valor (4,26 MPa) y Guaduas Cundinamarca el mayor valor con (12,67 MPa), ninguno de los resultados supera el valor registrado en la NSR-10, los valores de todos los resultados fueron corregidos con los coeficientes de modificación para contenido de humedad.

En la comparación de los módulos de elasticidad, la guadua del Tolima obtuvo el mayor valor $13.439 \mathrm{MPa}$. El menor valor lo obtuvo la guadua de Oiba-Santander, con un valor de 10409,778 MPa. El valor reportado por la NSR-10 es de $9500 \mathrm{MPa}$ como se observa en la figura 31.

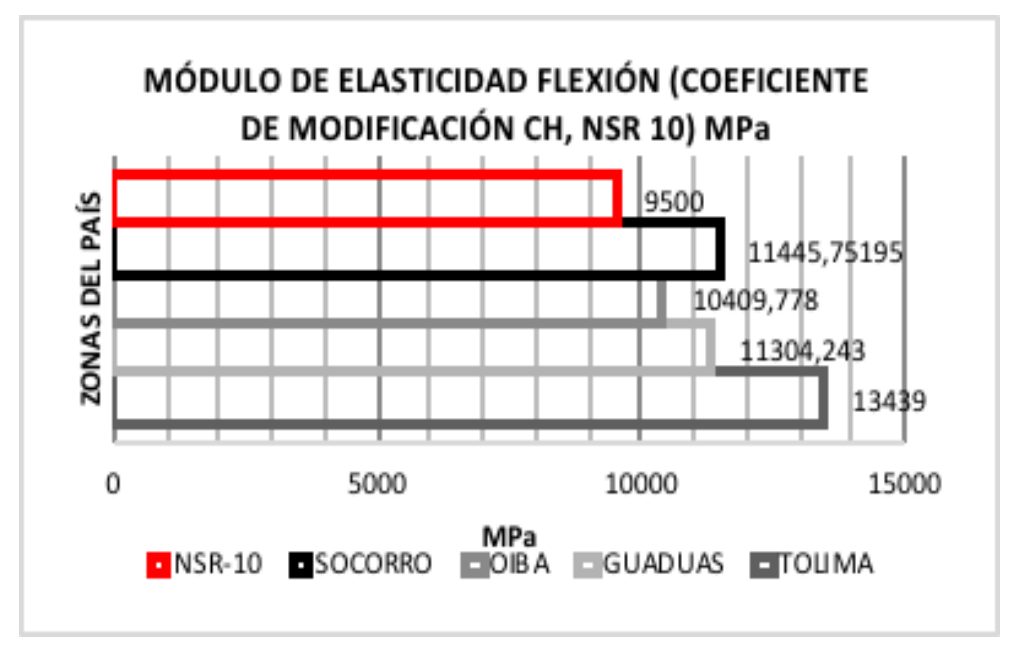

Figura 31. Comparación módulo de elasticidad flexión (coeficiente de modificación CH -NSR 10).

\subsection{ENSAYO A COMPRESIÓN PARALELA A LAS FIBRAS}

En comparación con los esfuerzos admisibles de Tolima, Guaduas-Cundinamarca y Oiba-Santander, Socorro Santander tiene el menor valor (2.27 MPa) y Tolima el mayor valor con (12.32 MPa), ninguno de los resultados supera el valor mínimo de la NSR-10 (14 MPa), los valores de todos los resultados fueron corregidos con los coeficientes de modificación para contenido de humedad.

En la comparación de los módulos de elasticidad Socorro Santander es el menor valor obtenido, el máximo valor lo obtuvo la guadua de Oiba-Santander, con 31059,36 MPa, como se observa en la figura 32.

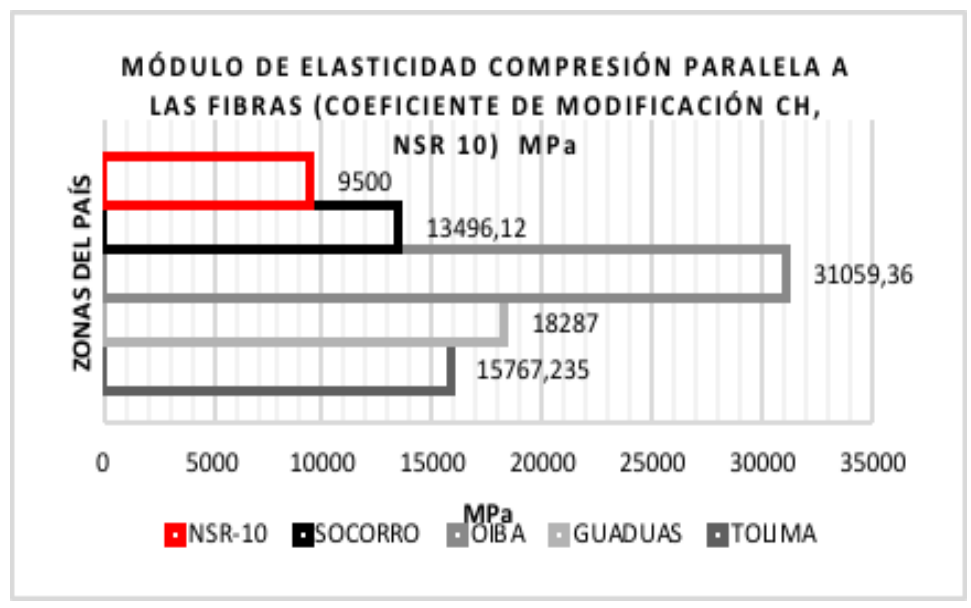

Figura 32. Comparación módulo de elasticidad compresión paralela a las fibras (coeficiente de modificación CH -NSR 10). 
Comparación de las propiedades físico-mecánicas del bambú Guadua angustifolia Kunth de diferentes municipios de Colombia

\subsection{ENSAYO A TENSIÓN PARALELA A LAS FIBRAS}

En la comparación de los esfuerzos admisibles Socorro Santander tiene el menor valor (3.03 MPa) y Guaduas el mayor valor con (4.85 MPa), ninguno de los resultados supera el valor mínimo de la NSR-10 (18 MPa), los valores de todos los resultados fueron corregidos con los coeficientes de modificación para contenido de humedad.

En la comparación entre los módulos de elasticidad, Oiba-Santander es el menor valor $8482.671 \mathrm{MPa}$, el máximo valor lo obtuvo la guadua de Guaduas-Cundinamarca, con $17537.64 \mathrm{MPa}$, como se observa en la figura 33.

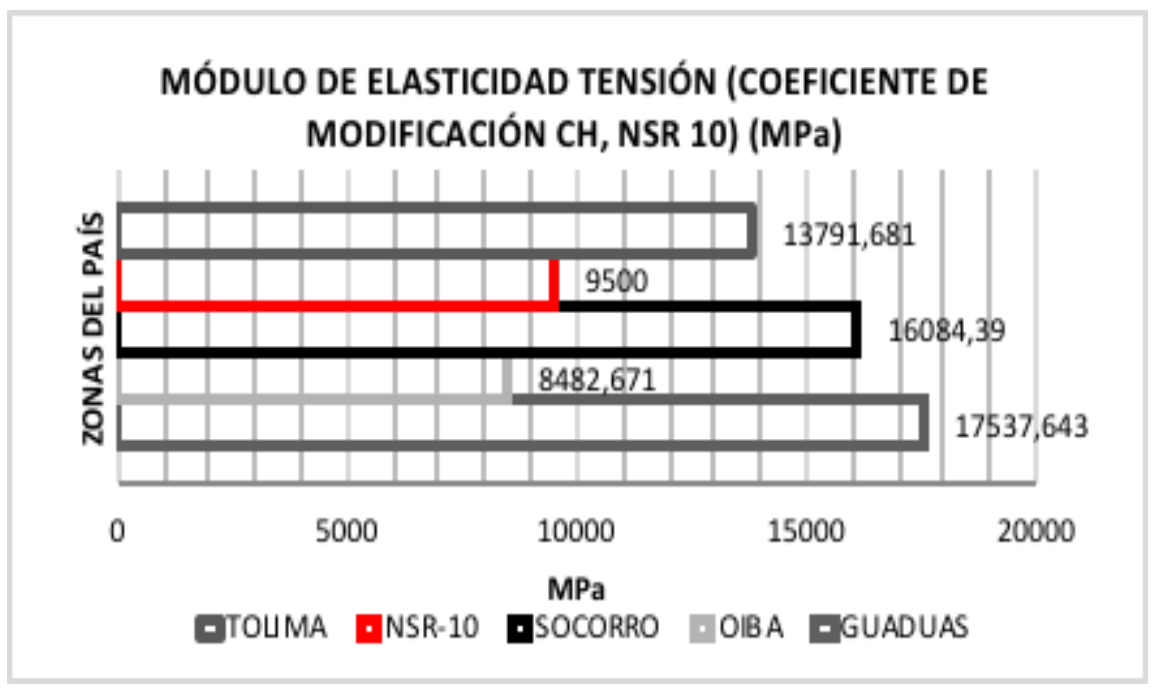

Figura 33. Comparación módulo de elasticidad tensión paralela a las fibras (factor de corrección CH -NSR 10).

\subsection{ENSAYO A COMPRESIÓN PERPENDICULAR A LAS FIBRAS}

En comparación con los esfuerzos admisibles de Tolima, Guaduas-Cundinamarca y Oiba-Santander, Oiba-Santander tiene el menor valor (0.66 MPa) y Guaduas el mayor valor con (1.89 MPa), Socorro-Santander ocupa el tercer lugar con 1.01 MPa.

\subsection{ENSAYO DE CORTE PARALELO A LAS FIBRAS}

En la comparación de los esfuerzos admisibles, Oiba-Santander tiene el menor valor (0.45 MPa) y Tolima el mayor valor con (1,31 MPa), Socorro-Santander ocupa el segundo lugar con 1,30 MPa. Los resultados de Socorro-Santander y Tolima son mayores que el valor especificado en la NSR-10 (1,40 MPa), los valores de todos los resultados fueron corregidos con los coeficientes de modificación para contenido de humedad.

\subsection{ENSAYO DE DENSIDAD}

Se realizó el ensayo de densidad siguiendo las indicaciones de la NTC 5525, tomando las medidas de espesores y secciones correspondientes, en la comparación de las investigaciones, se evidencia que el mayor valor es el de Guaduas- Cundinamarca $(620,41 \mathrm{~kg} / \mathrm{m} 3)$ y el menor es el de Oiba-Santander $(531,26 \mathrm{~kg} / \mathrm{m} 3)$. El resultado de Socorro $(545,98 \mathrm{~kg} / \mathrm{m} 3)$ se encuentra en la mitad, siendo un valor más cercano al de Oiba- Santander, estos municipios son limítrofes y es probable que por la similitud en cuanto a altitud y temperatura presenten algunos valores cercanos, Lo que evidencia que las condiciones del territorio donde crece la Guadua angustifolia Kunth afectan su estructura y por ende su comportamiento. 


\section{REFERENCIAS}

AIS. NSR-10 (Reglamento Colombiano De Construccion Sismoresistente). , (2010).

Ardila Pinilla, C. L. (2013). Determinación de los valores de esfuerzos admisibles del bambú Guadua angustifolia Kunth del departamento de Tolima, Colombia. 106. Retrieved from http://www.bdigital.unal.edu.co/12616/

Garzón Aponte, A. F. (2016). Caracterización físico-mecánica de la guadua en el municipio de Guaduas- Cundinamarca. 112.

Instituto Colombiano de Normas Técnicas ICONTEC. NTC 5525. , (2007).

MADR UNAL. (2010). Validación de la Guadua angustifolia como material estructural para diseño, por el método de esfuerzos admisibles.

Moreno, L. E., Osorio, L., \& Trujillo, E. E. (2006). Estudio de las propiedades mecánicas de haces de fibra de Guadua angustifolia. Ingeniería y Desarrollo, (20), 125-133.

Narváez Estefan, S. (2017). Caracterización de las propiedades físico - mecánicas de la Guadua angustifolia Kunth del municipio de Oiba, Santander. 129. Retrieved from http://www.bdigital.unal.edu.co/57366/

Rodríguez, W. (2007). Análisis del comportamiento de la producción y comercialización de la guadua y su efecto económico y social en el departamento de Santander. 1-69. Retrieved from http://repository.lasalle.edu.co/ bitstream/10185/12119/1/T10.07 R618a.pdf

Sánchez Escobar, L. C. (2018). Caracterización de las propiedades físico-mecánicas de la Guadua angustifolia Kunth del municipio de Socorro, Santander-Colombia (Universidad Nacional de Colombia). Retrieved from http://bdigital.unal.edu.co/71484/7/LauraCatalinaSánchezEscobar.2018.pdf

Takeuchi, C. P., \& González, C. E. (2007). Resistencia a la compresión paralela a la fibra de la Guadua angustifolia y determinación del módulo de elasticidad. Ingeniería y Universidad, 11(1), 89-103. https://doi.org/10.4067/ S0718-221X2001000100005

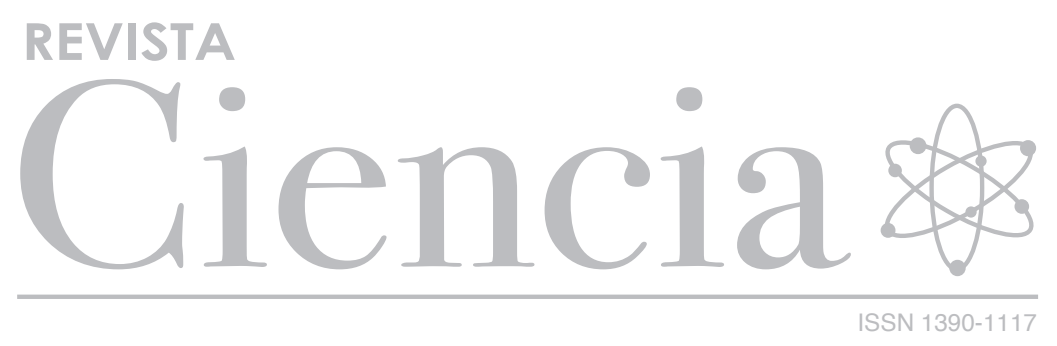

